\title{
ZERO-DIMINISHING LINEAR TRANSFORMATIONS
}

\author{
THOMAS CRAVEN ${ }^{1}$ AND GEORGE CSORDAS
}

\begin{abstract}
This paper solves an open problem of Karlin. We consider linear transformations defined on the set of all polynomials with real coefficients and give a complete characterization of the transformations with the property that they maintain or reduce the number of real zeros of each polynomial.
\end{abstract}

1. Introduction. Let $f$ be any real-valued function defined on $(-\infty, \infty)$. We shall use the notation $Z_{R}(f), Z_{+}(f)$ and $Z_{-}(f)$ for the number of real zeros of $f$, the number of zeros in $(0, \infty)$ and the number of zeros in $(-\infty, 0)$, respectively, counting multiplicities.

Let $T$ be a linear transformation defined on polynomials by $T\left(\sum_{k=0}^{n} a_{k} x^{k}\right)=$ $\sum_{k=0}^{n} a_{k} \lambda_{k} x^{k}$ with the eigenvalues $\lambda_{k}$ real, $k=0,1,2, \ldots$ In [3], Karlin raises the following question: When is a sequence $\lambda_{0}, \lambda_{1}, \lambda_{2}, \ldots$ a zero-diminishing sequence; that is, for which transformations $T$ does $Z_{R}(T f)<Z_{R}(f)$ hold for all polynomials $f$ ? As a special case, Pólya [8] and Karlin [3, p. 379] characterize the entire functions $\psi(z)$ for which $\lambda_{k}=1 / \psi(k)$ has this property. This result is based on the following theorem of Laguerre [7, p. 6].

THEOREM 1.1 (LAGUERRE). Let $Q(x)$ be a polynomial, all of whose zeros are real and negative. Let $F(x)=\Sigma a_{k} x^{k}$ be an arbitrary polynomial. Then

$$
Z_{R}\left(\sum a_{k} Q(k) x^{k}\right)>Z_{R}\left(\sum a_{k} x^{k}\right) \text {. }
$$

For the various analogues and extensions of Theorem 1.1 we refer the reader to [4], [6], [8] and [11]. In addition, an account of these important investigations is given by M. Marden [5].

One notes immediately from the proof of Theorem 1.1 (see, for example, [7, p. 6]) that $Z_{R}$ can be replaced by $Z_{+}$or $Z_{-}$or, in fact, by the number of zeros in an interval $(0, a)$ for any positive number $a$.

EXAMPLE 1.2. When $Q(x)$ has degree 1 , this corresponds to applying the differential operator $\left(a+x D_{x}\right), a>0$. The corresponding zero-diminishing transformation $T$ is defined by $T\left(x^{k}\right)=x^{k} /(a+k)$; that is, the eigenvalues are $\lambda_{k}=(a+k)^{-1}$, $k=0,1,2, \ldots$.

Besides the class of zero-diminishing transformations arising in the above manner, a handful of special cases have been worked out by Pólya [8]. (For additional references and examples see Karlin [3] and Pólya and Szegö [10].) In the

Received by the editors January 3, 1980.

1980 Mathematics Subject Classification. Primary 12D10, 26C10; Secondary 30C15.

Key words and phrases. Zero-diminishing transformation, polynomial, number of real zeros, entire function, exponential polynomial.

${ }^{1}$ Partially supported by National Science Foundation grant MCS76-06310. 
following section we provide a complete characterization of such linear transformations.

2. The characterization. We begin by noting that if $T$ is a zero-diminishing transformation, then all of the eigenvalues $\lambda_{k}$ are nonzero. For if $\lambda_{k}=0$, then $T\left(x^{k}\right)=0$ has infinitely many zeros while $x^{k}$ has only $k$ including multiplicity.

THEOREM 2.1. Let the transformation $T$ be defined by $T\left(\sum_{k=0}^{n} a_{k} x^{k}\right)=$ $\sum_{k=0}^{n} a_{k} \lambda_{k} x^{k} ; \lambda_{k} \neq 0, k=0,1,2, \ldots$ The following statements are equivalent.

(i) Either (a) $Z_{+}(T f)<Z_{+}(f)$ for all polynomials $f$ or (b) $Z_{+}(T f)<Z_{-}(f)$ for all polynomials $f$.

(ii) $T$ is a zero-diminishing transformation; that is, $Z_{R}(T f)<Z_{R}(f)$ for all polynomials $f$.

(iii) For each integer $n$, the polynomial $\sum_{k=0}^{n}\left(\begin{array}{c}n \\ k\end{array}\right) \lambda_{k}^{-1} x^{k}$ has only real zeros, all of the same sign.

(iv) The series $\varphi(z)=\sum_{k=0}^{\infty}\left(k ! \lambda_{k}\right)^{-1} z^{k}$ converges in the whole complex plane and the entire function $\varphi(z)$ can be represented in the form $\varphi(z)=c e^{0 z} \prod_{n=1}^{\infty}\left(1+z / z_{n}\right)$ where $\sigma>0, z_{n}>0, c$ is real and $\sum_{n=1}^{\infty} z_{n}^{-1}<\infty$.

Furthermore, in case (i)(a) all of the numbers $\lambda_{k}$ have the same sign and in case (i)(b) they alternate in sign.

Proof. (i) $\Rightarrow$ (ii). If (i)(a) holds, then let $g(x)=f(-x)$. Since $Z_{+}(T g)<Z_{+}(g)$, we have $Z_{-}(T f)<Z_{-}(f)$. The polynomials $T f$ and $f$ have the same multiplicity of the origin as a root and $Z_{+}(T f)<Z_{+}(f)$ by hypothesis. Thus $Z_{R}(T f)<Z_{R}(f)$. If (i)(b) holds, we again set $g(x)=f(-x)$, obtaining $Z_{-}(T f)=Z_{+}(T g)<Z_{-}(g)=$ $Z_{+}(f)$, which as before leads to $Z_{R}(T f)<Z_{R}(f)$.

(ii) $\Rightarrow$ (iii). In particular, if $T f$ is a polynomial with only real zeros, the polynomial $f$ must also have only real zeros. Equivalently, for any polynomial $f(x)=$ $\sum a_{k} x^{k}$ with only real zeros, the polynomial $T^{-1} f(x)=\sum a_{k} \lambda_{k}^{-1} x^{k}$ also has only real zeros. Thus for any positive integer $n, T^{-1}(x+1)^{n}=\sum_{k=0}^{n}\left(\begin{array}{l}n \\ k\end{array}\right) \lambda_{k}^{-1} x^{k}$ has only real zeros. Considering $T^{-1} x^{k}(x+1)(x-1)=\left(\lambda_{k}^{-1}-\lambda_{k+2}^{-1} x^{2}\right) x^{k}$, we see that the numbers $\lambda_{k}$ and $\lambda_{k+2}$ must have the same sign. If all $\lambda_{k}$ have the same sign, the roots of $\Sigma\left(\begin{array}{l}n \\ k\end{array}\right) \lambda_{k}^{-1} x^{k}$ are all negative, and if the $\lambda_{k}$ alternate in sign, the roots are all positive.

(iii) $\Leftrightarrow$ (iv). This equivalence is a classical result of Pólya and Schur [9].

(iii) $\Rightarrow$ (i). Assume $g_{m}(x)=\sum_{k=0}^{m}\left(\begin{array}{c}m \\ k\end{array}\right) \lambda_{k}^{-1} x^{k}$ has only real zeros, all of the same sign. Then all of the $\lambda_{k}$ have the same sign or they alternate in sign. Without loss of generality, we may assume that the zeros of $g_{m}(x)$ are all negative and thus the $\lambda_{k}$ all have the same sign. Otherwise, let $\mu_{k}=(-1)^{k} \lambda_{k}$ and $S$ be the transformation defined by the $\mu_{k}$, which all have the same sign. If we know $Z_{+}(S f(x))<Z_{+}(f(x))$, then we obtain

$$
Z_{+}(T f(-x))=Z_{+}(S f(x))<Z_{+}(f(x))=Z_{-}(f(-x)),
$$

thus completing the second case since the polynomial $f$ is arbitrary. Clearly we may also assume $\lambda_{k}>0$ for each $k$.

Now let $f(x)=\sum_{k=0}^{n} a_{k} x^{k}$ be an arbitrary polynomial of degree $n$, and consider the polynomial $g_{m}(x)=\sum_{k=0}^{m}\left(\begin{array}{c}m \\ k\end{array}\right) \lambda_{k}^{-1} x^{k}, m>n$, which has only real negative zeros. 
In [2, Corollary 3.6], we have generalized a classical theorem of Schur [6, Satz 7.4] to arbitrary polynomials $f$. This result states that $Z_{+}\left(\sum_{k=0}^{n} k ! a_{k}\left(\begin{array}{c}m \\ k\end{array}\right) \lambda_{k}^{-1} x^{k}\right)>Z_{+}(f)$. Replacing $x$ by $x / m$ gives

$$
Z_{+}\left(\sum_{k=0}^{n} a_{k} \lambda_{k}^{-1} \frac{m !}{(m-k) ! m^{k}} x^{k}\right)>Z_{+}(f),
$$

for all $m \geqslant n$. We note that $\lim _{m \rightarrow \infty} m ! /(m-k) ! m^{k}=1$ for each fixed $k$. By Hurwitz's theorem, taking the limit as $m$ approaches infinity cannot decrease the left-hand side of (2.2). Therefore we obtain

$$
Z_{+}\left(\sum_{k=0}^{n} a_{k} \lambda_{k}^{-1} x^{k}\right)>Z_{+}(f)
$$

or equivalently, $Z_{+}(f)>Z_{+}(T f)$, thus concluding the proof of the theorem.

Replacing the variable $x$ by $e^{x}$ in part (i)(a) of the theorem gives the following result on exponential polynomials.

COROllary 2.3. Let $\lambda_{0}, \lambda_{1}, \lambda_{2}, \ldots$ be a zero-diminishing sequence with all $\lambda_{k}>0$. For any real numbers $a_{0}, a_{1}, \ldots, a_{n}$, we have

$$
Z_{R}\left(\sum_{k=0}^{n} a_{k} \lambda_{k} e^{k x}\right)<Z_{R}\left(\sum_{k=0}^{n} a_{k} e^{k x}\right) .
$$

We note that this corollary is closely related to results of Benz [1, Satz 7, p. 273].

REMARK 2.4. There is still an open problem regarding finite sequences $\lambda_{0}, \lambda_{1}, \ldots, \lambda_{n}$ which are zero-diminishing for all polynomials of degree at most $n$. Our result leads only to the sequences given by part (iv) of Theorem 2.1: either they are part of a longer sequence or the polynomial $\sum_{k=0}^{n}\left(k ! \lambda_{k}\right)^{-1} x^{k}$ must have only real zeros, all of the same sign. One can construct examples to show that other finite zero-diminishing sequences also exist which do not correspond to the initial portion of an entire function in the class considered in (iv) of Theorem 2.1.

\section{REFERENCES}

1. E. Benz, Über lineare, verschiebungstreue Funktionaloperationen und die Nullstellen ganzer Funktionen, Comment. Math. Helv. 7 (1934), 243-289.

2. T. Craven and G. Csordas, On the number of real roots of polynomials (to appear).

3. S. Karlin, Total positivity. Vol. 1, Stanford Univ. Press, Stanford, Calif., 1968.

4. M. Marden, The zeros of certain composite polynomials, Bull. Amer. Math. Soc. 49 (1943), 93-100.

5. _ Geometry of polynomials, 2nd ed., Math. Surveys, no. 3, Amer. Math. Soc., Providence, R.I., 1966.

6. N. Obreschkoff, Sur les zéros des polynômes, C. R. Acad. Sci. Paris 208 (1939), 1270-1272.

7. __ Verteilung und Berechnung der Nullstellen reeler Polynome, VEB Deutscher Verlag, Berlin, 1963.

8. G. Pólya, Über einen Satz von Laguerre, Jber. Deutsch. Math.-Verein. 38 (1929), 161-168.

9. G. Pólya and J. Schur, Über zwei Arten von Faktorenfolgen in der Theorie der algebraischen Gleichungen, J. Reine Angew. Math. 144 (1914), 89-113.

10. G. Pólya and G. Szegö, Aufgaben und Lehrsätze aus der Analysis. I, II, 2nd ed., Springer-Verlag, Berlin, 1954; English transl., 1975.

11. L. Weisner, Roots of certain classes of polynomials, Bull. Amer. Math. Soc. 48 (1942), 283-286.

Department of Mathematics, University of Hawail, Honolulu, Hawall 96822 